\title{
Milk fat globule E-8 and interleukin 17 in systemic lupus erythematosus: partners in crime?
}

\author{
Lamiaa Madkour, Fatema Elgengehy, Marwa Niazy, Shada Ghoneim \\ Faculty of Medicine, Cairo University, Egypt
}

\begin{abstract}
Objectives: Systemic lupus erythematosus (SLE) is a multi-factorial, autoimmune disease with a wide array of manifestations. The pro-inflammatory cytokine interleukin (IL)-17 has been implicated in the inflammatory response and tissue damage in SLE; however, its correlation with disease activity is still questionable. Meanwhile, efficient clearance of apoptotic cells is required for immune tolerance. An abnormally low or high level of milk fat globule (MFG-E8) can result in impaired apoptotic cell clearance and the subsequent autoimmune response. In this study, we endeavoured to compare the levels of MFG-E8 and IL-17 in SLE patients and healthy controls and to reveal the alleged association of these levels with SLE disease activity.

Material and methods: Serum samples from 57 SLE patients and 30 healthy control subjects were examined for quantitation of MFG-E8 and IL-17 levels using ELISA. Systemic lupus erythematosus disease activity was calculated using the SLE Disease Activity Index (SLEDAI). Clinical manifestations and laboratory findings of the patients were also recorded.

Results: We report that serum MFG-E8 levels were significantly elevated in the sera of SLE patients compared to healthy controls $(p$-value $=0.019)$. Likewise, IL-17 levels were higher in SLE patients ( $p$-value $<0.001)$. A positive correlation was revealed between MFG-E8 level and proteinuria. Surprisingly, there was a poor correlation between disease activity and the levels of either IL-17 or MFG-E8.

Conclusions: Although serum MFG-E8 and IL-17 levels were higher in SLE patients than in normal controls, our results indicate that they cannot accurately reflect the disease activity. Meanwhile, further studies are needed to assess MFG-E8 and IL-17 as potential therapeutic targets in SLE patients.
\end{abstract}

Key words: apoptosis, autoimmune diseases, MFG-E8, systemic lupus erythematosus.

\section{Introduction}

Systemic lupus erythematosus is an autoimmune disease affecting around 0.2 to 2 per 1000 individuals [1], with a female to male ratio of 9 to 1 [2]. The disease is chronic, with alternating periods of flare and remission. It can affect multiple organs, including the joints, kidneys, skin, cardiovascular system and nervous system. The pathogenesis of the disease is believed to be multifactorial. In addition to the complex interaction between genetic and environmental factors, the pathogenesis also involves autoantibody formation and immune complex deposition, as well as tissue infiltration by lymphocytes [3].

Due to the high level of autoantibodies, SLE has traditionally been considered to be B-cell dependent; however, in recent years, it has become clear that T cells have a major role in the pathogenesis of this disease [4].

It has been demonstrated that in SLE patients, T cells exhibit certain biochemical and functional abnormalities that affect their activation process, cytokine production and expression of co-stimulatory molecules [5]. A subset of T-helper cells that produces interleukin 17 (IL-17), Th17

Address for correspondence:

Lamiaa Madkour, Faculty of Medicine, Cairo University, Kasralainy Str., 11211 Cairo, Egypt, e-mail: m_l@kasralainy.edu.eg

Submitted: 14.10.2015; Accepted: 9.01.2016 
has been implicated in the pathogenesis of systemic autoimmune diseases including SLE and psoriasis, as well as rheumatoid arthritis [6].

Th17 cells differentiate from naive $T$ cells following T-cell receptor (TCR) activation and co-stimulation in the presence of transforming growth factor $\beta$ (TGF- $\beta$ ) and IL-6 [7]. In addition, IL-23 has a major role in their proliferation and terminal differentiation [8]. Besides Th17 cells, IL-17 is also produced by $\gamma \delta$-T cells, NK cells, neutrophils [9] and CD4-CD8- double-negative (DN) T cells [10].

Interleukin 17 is a pro-inflammatory cytokine that contributes to the defence against certain pathogens, mainly extracellular bacteria and fungi [9]. In addition, IL-17 induces tissue inflammation via stimulation of proinflammatory cytokines, e.g. IL-6, tumour necrosis factor $\alpha$ (TNF- $\alpha$ ), and IL-1 $\beta$ [11], and in turn synergizes with them to induce multiple inflammatory factors. Moreover, IL-17 has been shown to synergize with B-cell-activating factor (BAFF) to mediate B-cell survival, thereby promoting the number of autoantibody-producing cells [12].

In addition to its potent pro-inflammatory activity, IL-17 recruits neutrophils and monocytes by increasing the production of chemokines, including IL-8 and monocyte chemo-attractant protein-1 [13]. Interleukin 17 also facilitates T-cell infiltration and activation through stimulating the expression of intracellular adhesion molecule-1 [14] as well as the production of IL- 6 and granulocyte-monocyte-colony stimulating factor [15].

Further supporting the presence of a Th17-biased environment in patients with SLE, increased plasma levels of IL-6, a crucial differentiating factor for Th17 cells, as well as IL-21, a Th17 cytokine, have been observed in SLE patients [16].

Of note, some studies were carried out to comprehend the genetic basis of SLE. A study conducted in Italy [17] revealed a significant association between TRAF3IP2 gene polymorphism and susceptibility to SLE. The TRAF3IP2 gene codes for Act1, which is a positive signal adaptor for IL-17-mediated immune responses, and in the meantime it negatively regulates acquired immunity. Another study by Ciccacci et al. [18] verified that polymorphisms in 3 genes (STAT4, IL-10, and HCP5) were risk factors for the development of SLE. However, such polymorphisms contribute by only a small fraction to the overall genetic susceptibility, which still remains enigmatic.

Meanwhile, another potential trigger of SLE is the impaired clearance of apoptotic cells. In fact, apoptosis is a critical process in immune tolerance and in maintenance of normal immune responses to foreign antigens [19].

The efficient recognition and clearance of apoptotic cells involve many receptors, ligands and bridging molecules between dying cells and phagocytes [20].
Early apoptotic cells express eat-me signals through asymmetric distribution of amino-phospholipids such as phosphatidylserine (PS), phosphatidylethanolamine and phosphatidylcholine. Phosphatidylserine is the best-characterized eat-me signal, and externalization of PS alone is sufficient to initiate the engulfment of apoptotic cells by macrophages [21]. In addition, apoptotic cells actively secrete chemoattractant factors known as find-me signals to attract phagocytes.

In the germinal centres, clonal selection of high-affinity B cells results in large numbers of apoptotic cells, including auto-reactive cells. Clearance of these cells is critical to the maintenance of peripheral tolerance. Otherwise, persistence of apoptotic cells may progress to secondary necrotic cell death. This, in turn, is associated with accessible modified auto-antigens and initiation of the autoimmune response [21].

MFG-E8 is a glycoprotein that plays an important role in the clearing of apoptotic cells. It is secreted by activated macrophages or immature dendritic cells and acts as a bridging molecule between apoptotic cells and phagocytes, which promotes engulfment of apoptotic cells [22]. It is reported that there is an optimal concentration of MFG-E8 to promote phagocytosis. The activity of phagocytosis increases dose dependently at a low concentration, but above the optimal concentration the effect changes toward inhibition. This can be explained by the hypothesis that excess MFG-E8 saturates the binding sites on apoptotic cells and phagocytes, and thus cannot work as a bridging molecule [23]. In this way, an excess as well as a deficiency of MFG-E8 causes impairment of apoptotic cell clearance and may induce autoimmune disease [22].

In this study, we provide evidence that the serum levels of IL-17 as well as of MFG-E8 are higher in SLE patients than in healthy control participants.

\section{Material and methods \\ Participants and clinical assessments}

Fifty-seven SLE patients and 30 normal control subjects were included in this study. The 57 SLE patients were attending the outpatient clinic or admitted to the inpatient unit of the Rheumatology and Rehabilitation Department, Cairo University Hospitals, during a 6-month-period, from November 2013 to April 2014. The enrolled patients fulfilled the American College of Rheumatology (ACR) revised criteria [24].

Demographic characteristics, clinical features and laboratory findings (CBC, ESR, ANA, anti-dsDNA, ACL, C3, $C 4, A L T$, renal function tests, urine analysis and 24-hour urinary protein) of the patients were recorded. Renal biopsy was done for patients with nephritis. Disease-related damage was recorded using the Systemic Lupus 
International Collaborating Clinics (SLICC) SLE Damage Index (SDI). Disease activity was recorded using the SLE Disease Activity Index (SLEDAI).

Thirty healthy participants, matched for age and gender, served as a control group. All participants were informed about the study aims and protocol, and informed consent was obtained prior to the study. The study obtained the consent of the bioethics committee.

\section{Samples}

Serum samples were collected from patients at routine medical visits. Sera were stored at $-20^{\circ} \mathrm{C}$ until use. Sera of the control group were collected from 30 healthy volunteers.

\section{Measurement of serum MFG-E8}

Serum MFG-E8 levels were measured by ELISA as per the manufacturer's instructions. First, the serum samples were diluted $1: 6$ in Calibrator Diluent RD5-20. Following the addition of Assay Diluent RD1-57 to the wells, $50 \mathrm{ml} /$ well of sample, standard or control was added and incubated for 2 hours at room temperature on a microplate shaker. The wells were then washed 4 times. Thereafter, $200 \mu$ l of MFG-E8 conjugate was added to each well and incubated for 2 hours at room temperature on a microplate shaker. The wells were washed 4 times again, and incubated with $50 \mathrm{ml} /$ well of Substrate Solution for 30 minutes at room temperature. Thereafter, the stop solution was added and the absorbance values were determined at $450 \mathrm{~nm}$. The results were expressed as picograms per millilitre $(\mathrm{pg} / \mathrm{ml})$.

\section{Measurement of serum interleukin 17}

Serum IL-17 levels were measured by quantitative sandwich ELISA using the Human IL-17 ELISA Kit ( $R$ \& D Systems, Minneapolis, MN, USA). The wells had been pre-coated with anti-IL-17 monoclonal antibody (mAb). First, the Assay Diluent RD1-36 was added (100 $\mathrm{\mu l} /$ well). Then, $100 \mu \mathrm{l} /$ well of serum samples, control or standard was added followed by incubation for 4 hours at room temperature. After washing, $200 \mu \mathrm{l}$ of IL-17 conjugate was added to each well and incubated for 1 hour at room temperature. After washing and aspiration, the substrate solution was added to the wells and incubated for 30 minutes at room temperature. After that, the stop solution was added and the absorbance values were determined at $450 \mathrm{~nm}$. The results were expressed as pg/ml.

\section{Statistical analysis}

Data were analyzed using IBM SPSS advanced statistics version 20 (SPSS Inc., Chicago, IL). Numerical data were expressed as mean and standard deviation or median and range as appropriate. Qualitative data were expressed as frequency and percentage. Chisquare test or Fisher's exact test was used to examine the relation between qualitative variables. For quantitative data, comparison between two groups was done using either Student's t-test or the Mann-Whitney test (non-parametric $t$-test) for non-normally distributed numerical data. Spearman's rho method was used to test the correlation between numerical variables. The receiver operating characteristic (ROC) curve (Fig. 1) was used for prediction of cut-off values for the markers. All tests were two-tailed. A $p$-value $<0.05$ was considered significant.

\section{Results}

Fifty-seven SLE patients and 30 normal control participants were included in this study. Out of the $57 \mathrm{SLE}$ patients, $94.7 \%$ were females, while out of the 30 control subjects, $93.3 \%$ were females. The median disease duration in our patients was 7 years (mean \pm SD $=7.4$ \pm 5 .3). Demographic data of SLE patients and healthy control participants are shown in Table I.

The median SLEDAI of our SLE patients was 12 with a range of 1 to 35. Twenty-eight of our patients had mild disease activity, 19 had moderate activity and 10 were severely active (Table II).

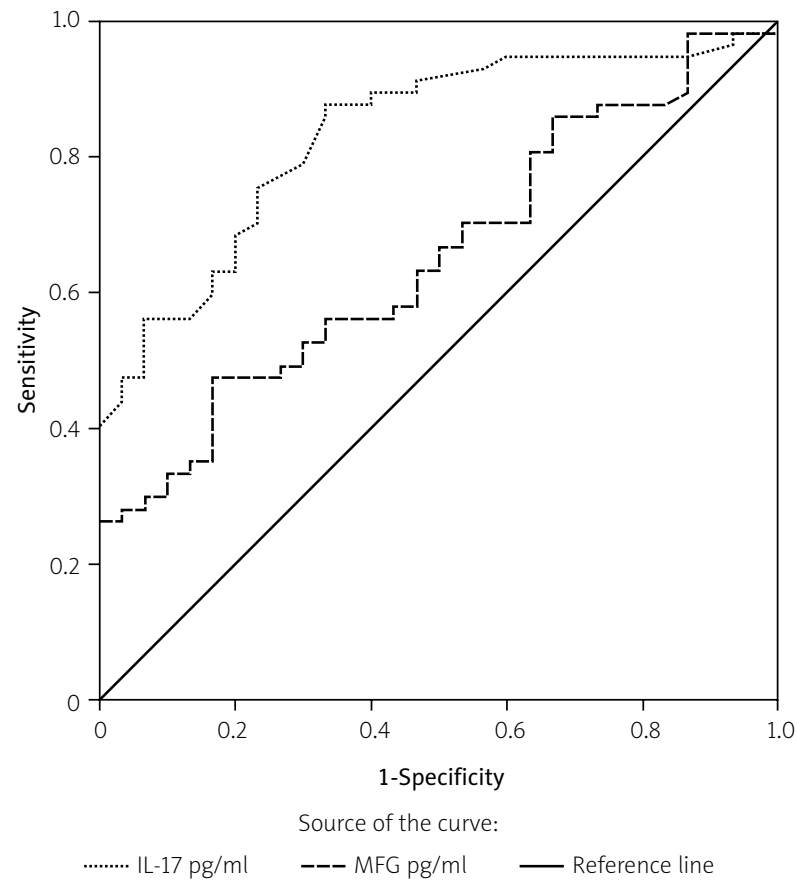

Fig. 1. Receiver operating characteristic (ROC) curve showing sensitivity and specificity of IL-17 and MFG-E8. 
Table I. Demographic data of SLE patients and healthy participants

\begin{tabular}{|lcccc|}
\hline Parameter & & Patients $(n=57)$ & Control $(n=30)$ & $P$-value \\
\hline Age (years) & range & $15-55$ & $28-50$ & 0.8 \\
\cline { 2 - 4 } & median & 29 & 28 & \\
\cline { 2 - 4 } & mean \pm SD & $29.9 \pm 8.2$ & $29.5 \pm 7.4$ & \\
\hline Gender (female/male) & & $54 / 3$ & $28 / 2$ & 1 \\
\hline
\end{tabular}

Table II. Clinical and laboratory findings in the 57 SLE patients

\begin{tabular}{|c|c|c|}
\hline Finding & & $\begin{array}{c}\text { Number (\%) } \\
\text { of patients }\end{array}$ \\
\hline \multirow[t]{3}{*}{ Disease severity } & mild & $28(49.1 \%)$ \\
\hline & moderate & $19(33.3 \%)$ \\
\hline & severe & $10(17.5 \%)$ \\
\hline \multirow[t]{7}{*}{ Clinical findings } & mucocutaneous lesions & $48(84.2 \%)$ \\
\hline & nephritis & $37(64.9 \%)$ \\
\hline & vasculitis & $11(19.3 \%)$ \\
\hline & serositis & $8(14 \%)$ \\
\hline & CNS manifestations & $6(10.5 \%)$ \\
\hline & fever & $3(5.3 \%)$ \\
\hline & arthritis & $1(1.8 \%)$ \\
\hline \multirow{10}{*}{$\begin{array}{l}\text { Laboratory } \\
\text { findings }\end{array}$} & positive ANA & $52(91.2 \%)$ \\
\hline & $\begin{array}{l}\text { positive anti-DNA } \\
\text { antibody }\end{array}$ & $41(71.9 \%)$ \\
\hline & anaemia & $24(42.1 \%)$ \\
\hline & leukopenia & $5(8 \%)$ \\
\hline & thrombocytopenia & $6(10.5 \%)$ \\
\hline & proteinuria & $32(56.1 \%)$ \\
\hline & pyuria & $23(40.4 \%)$ \\
\hline & haematuria & $11(19.3 \%)$ \\
\hline & $\begin{array}{c}\text { hypocomplementaemia } \\
\text { (C3) }\end{array}$ & $19(33.3 \%)$ \\
\hline & $\begin{array}{c}\text { hypocomplementaemia } \\
\text { (C4) }\end{array}$ & $6(10.5 \%)$ \\
\hline
\end{tabular}

CNS - central nervous system; ANA - anti-nuclear antibody

Among the 57 patients included in this study, the most frequent clinical variables at the time of sample collection were mucocutaneous manifestations (48 patients $=84.2 \%)$ and nephritis (64.9\%) followed by vasculitis (19.3\%), as shown in Table II.

\section{Serum interleukin 17 and MFG-E8 levels}

There were no differences in serum IL-17 or MFG-E8 levels among SLE patients according to gender. On the other hand, serum IL-17 levels were significantly higher in SLE patients than in the normal control group ( $p$-val- ue $<0.001)$. Likewise, there was a significant difference in MFG-E8 levels between SLE patients and the normal control group (Table III).

We also endeavoured to detect the association of IL-17 and MFG-E8 levels with disease activity. However, no correlation was detected between serum IL-17 or MFG-E8 levels and SLE disease activity assessed by SLEDAI (Figs. 2 and 3).

No correlation was detected between serum IL-17 or MFG-E8 levels and SLICC. Also, there was no association between serum IL-17 or MFG-E8 levels and anti-dsDNA antibodies, ESR, C3 or C4. Nonetheless, a significant association was found between elevated MFG-E8 and proteinuria > $3.5 \mathrm{gm} / 24 \mathrm{~h}(p$-value $=0.026)$.

\section{Discussion}

The role of IL-17 in the pathogenesis of SLE is well accepted, and can render it a potential therapeutic target [19]. Also, IL-17-producing T cells have been detected in the main target organs of SLE including kidneys, lungs, and skin, suggesting that IL-17 may be involved in the inflammatory response and subsequent tissue damage [25]. In line with expectations, this study revealed that IL-17 levels were significantly higher in SLE patients than in healthy control subjects. However, our study did not reveal an association between IL-17 levels and SLEDAI score, suggesting that IL-17 level may not accurately reflect the actual disease activity. Previous studies reported inconsistent results regarding this association. On one hand, Doreau et al. [12] reported a positive correlation between IL-17 level and SLEDAI; on the other hand, various studies [26] did not show any correlation between IL-17 and SLEDAI.

It is difficult to specify the reason behind the discrepancy in results among different studies. However, it may be attributed to variations of ELISA sensitivity, as well as the presence of confounding factors (e.g. immunosuppressive drugs) that influence serum IL-17 level. Moreover, serum IL-17 level may not be an accurate indicator of IL-17 production, as IL-17 may be localized to the inflamed tissues. Clinical trials targeting IL-17 are under way in several autoimmune diseases; e.g. secukinumab (AIN457), a human anti-IL-17 IgG1א monoclonal 
Table III. Comparison of IL-17 and MFG-E8 levels between SLE patients and the control group

\begin{tabular}{|lccccccc|}
\hline Parameter in $\mathrm{pg} / \mathrm{ml}$ & Mean & $\begin{array}{c}\text { Standard } \\
\text { deviation }\end{array}$ & Median & Minimum & Maximum & $P$-value \\
\hline IL-17 & patients & 27.8 & 11.6 & 26 & 7 & 66.5 & $<0.001$ \\
\cline { 2 - 7 } & controls & 17.1 & 4.7 & 15.5 & 10.8 & 27 & \multirow{2}{*}{0.019} \\
\hline \multirow{2}{*}{ MFG-E8 } & patients & 962.4 & 912.8 & 585 & 12.5 & 2940 & 1890 \\
\cline { 2 - 7 } & controls & 449.5 & 403.4 & 342.6 & 54 & \\
\hline
\end{tabular}

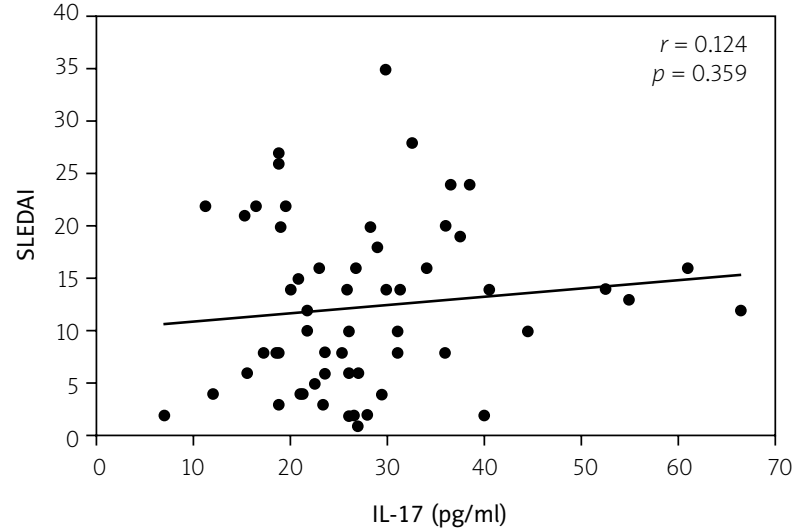

Fig. 2. Correlation between IL-17 levels and SLEDAI. $p$ - $p$-value; $r$-correlation coefficient

antibody, has been studied in rheumatoid arthritis, psoriasis and Crohn's disease. At present, no clinical trials of anti-IL-17 therapy are under way in SLE, but it appears to be a promising option [27].

Apoptotic cell clearance is a daunting challenge to the immune system. In animal models, it has been established that this process may fall short under certain conditions, resulting in persistent potentially immunogenic necrotic and apoptotic cells. Both in vivo and in vitro studies support the notion that SLE may also result from impaired apoptotic cell clearance [20].

Hence, the pro-inflammatory activity of IL-17 and its impact on B-cells can justify its role in the pathogenesis of SLE [28]. Accordingly, various correlations between serum IL-17 levels and SLE disease activity have been reported in previous studies [12]. Nevertheless, there is a dearth of data concerning the potential relationship between IL-17 and the clinical findings in SLE patients [27].

Our study revealed significantly higher levels of MFG-E8 in SLE patients compared to healthy control subjects; however, we found no correlation between MFG-E8 and disease activity. In a study by Yamaguchi et al. [23], high levels of MFG-E8 were observed in SLE patients, while the levels were undetectable in healthy controls. In addition, Yamamato et al. [29] found high levels of MFG-E8 in SLE patients, and these levels correlated with disease activity.

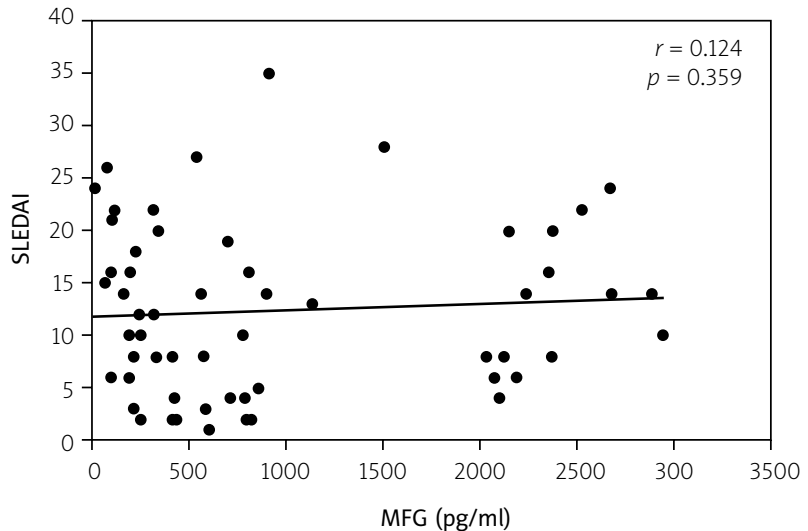

Fig. 3. Correlation between MFG-E8 levels and SLEDAI.

$p$-p-value; $r$-correlation coefficient

With excessive amounts of MFG-E8, the apoptotic cells may not be efficiently removed. The subsequent leakage of autoantigens may trigger a strong autoimmune response including the production of autoantibodies, deposition of immune complexes and complement consumption [29].

\section{Conclusion}

In conclusion, in the present study neither IL-17 nor MFG-E8 was found to be associated with SLE activity. But our study revealed significantly higher levels of MFG-E8 in SLE patients compared to healthy control subjects. However, it is also possible that the high MFG-E8 level is a secondary effect of inflammation or immune system activation in SLE [23]. Moreover, the hormonal imbalance in SLE patients could be another possible cause, because it was reported that prolactin can up-regulate MFG-E8 expression in macrophages and enhance the engulfment of apoptotic cells [30]. Hence, the mechanisms behind MFG-E8 elevation could be multifactorial, and further studies are needed. A better understanding of the immune biology of apoptotic cell clearance may lead to new therapeutic pipelines.

The authors declare no conflict of interest. 


\section{References}

1. Borchers AT, Naguwa SM, Shoenfeld Y, Gershwin ME. The geoepidemiology of systemic lupus erythematosus. Autoimmun Rev 2010; 9: A277-A287.

2. Truchetet ME, Mossalayi MD, Boniface K. IL-17 in the rheumatologist's line of sight. BioMed Res Int 2013; Article ID 295132

3. Adalid-Peralta L, Mathian A, Tran T, et al. Leukocytes and the kidney contribute to interstitial inflammation in lupus nephritis. Kidney Int 2008; 73: 172-180.

4. Hofstetter HH, Ibrahim SM, Koczan D, et al. Therapeutic efficacy of IL-17 neutralization in murine experimental autoimmune encephalomyelitis. Cell Immunol 2005; 237: 123-130.

5. Crispin JC, Kyttaris VC, Juang YT, Tsokos GC. How signalling and gene transcription aberrations dictate the systemic lupus erythematosus T cell phenotype. Trends Immunol 2008; 29: 110-115.

6. Ambrosi A, Espinosa A, Wahren-Herlenius M. IL-17: A new actor in IFN-driven systemic autoimmune diseases. Eur J Immunol 2012; 42: 2274-2284.

7. Bettelli E, Carrier Y, Gao W, et al. Reciprocal developmental pathways for the generation of pathogenic effector TH17 and regulatory T cells. Nature 2006; 441: 235-238.

8. Langrish CL, Chen Y, Blumenschein WM, et al. IL-23 drives a pathogenic $T$ cell population that induces autoimmune inflammation. J Exp Med 2005; 201: 233-240.

9. Korn T, Oukka M, Kuchroo V, Bettelli E. Th17 cells: effector T cells with inflammatory properties. Semin Immunol 2007; 19: 362-371.

10. Crispin JC, Oukka M, Bayliss G, et al. Expanded double negative $T$ cells in patients with systemic lupus erythematosus produce IL-17 and infiltrate the kidneys. J Immunol 2008; 181: 8761-8766.

11. Jovanovic DV, Di Battista JA, Martel-Pelletier J, et al. IL-17 stimulates the production and expression of proinflammatory cytokines, IL- $\beta$ and TNF- $\alpha$, by human macrophages. J Immunol 1998; 160: 3513-3521.

12. Doreau A, Belot A, Bastid J, et al. Interleukin 17 acts in synergy with $B$ cell-activating factor to influence $B$ cell biology and the pathophysiology of systemic lupus erythematosus. Nat Immunol 2009; 10: 778-785.

13. Agarwal S, Misra R, Aggarwal A. Interleukin 17 levels are increased in juvenile idiopathic arthritis synovial fluid and induce synovial fibroblasts to produce proinflammatory cytokines and matrix metalloproteinases. J Rheumatol 2008; 35: 515-519.

14. Albanesi C, Cavani A, Girolomoni G. IL-17 is produced by nickelspecific T lymphocytes and regulates ICAM-1 expression and chemokine production in human keratinocytes: synergistic or antagonist effects with IFN-gamma and TNF-alpha. J Immunol 1999; 162: 494-502.

15. Schwarzenberger P, Huang W, Ye P, et al. Requirement of endogenous stem cell factor and granulocyte-colony-stimulating factor for IL-17-mediated granulopoiesis. J Immunol 2000; 164: 4783-4789.

16. Shah K, Lee WW, Lee SH, et al. Dysregulated balance of Th17 and Th1 cells in systemic lupus erythematosus. Arthritis Res Ther 2010; 12: R53.
17. Perricone C, Ciccacci C, Ceccarelli F, et al. TRAF3IP2 gene and systemic lupus erythematosus: association with disease susceptibility and pericarditis development. Immunogenetics 2013; 65: 703-709.

18. Ciccacci C, Perricone C, Ceccarelli F, et al. A multilocus genetic study in a cohort of Italian SLE patients confirms the association with STAT4 gene and describes a new association with HCP5 gene. PLoS One 2014; 9: e111991.

19. Munoz LE, Lauber K, Schiller M, et al. The role of defective clearance of apoptotic cells in systemic autoimmunity. Nat Rev Rheumatol 2010; 6: 280-289.

20. Shao WH, Cohen PL. Disturbances of apoptotic cell clearance in systemic lupus erythematosus. Arthritis Res Ther 2011; 13: 202.

21. Fadeel B, Xue D, Kagan V. Programmed cell clearance: molecular regulation of the elimination of apoptotic cell corpses and its role in the resolution of inflammation. Biochem Biophys Res Commun 2010; 396: 7-10.

22. Hanayama R, Tanaka M, Miwa K, et al. Identification of a factor that links apoptotic cells to phagocytes. Nature 2002; 417: 182-187.

23. Yamaguchi H, Takagi J, Miyamae T, et al. Milk fat globule EGF factor 8 in the serum of human patients of systemic lupus erythematosus. J Leukoc Biol 2008; 83: 1300-1307.

24. Hochberg MC. Updating the American College of Rheumatology revised criteria for the classification of systemic lupus erythematosus. Arthritis Rheum 1997; 40: 1725-1725.

25. Wang Y, Ito S, Chino Y, et al. Laser microdissection - based analysis of cytokine balance in the kidneys of patients with lupus nephritis. Clin Exp Immunol 2010; 159: 1-10.

26. Zhao XF, Pan HF, Yuan H, et al. Increased serum interleukin 17 in patients with systemic lupus erythematosus. Mol Biol Rep 2010; 37: 81-85.

27. Vincent FB, Northcott $M$, Hoi A, et al. Clinical associations of serum interleukin-17 in systemic lupus erythematosus. Arthritis Res Ther 2013; 15: R97.

28. Zhu S, Qian Y. IL-17/IL-17 receptor system in autoimmune disease: mechanisms and therapeutic potential. Clin Sci 2012; 122: 487-511.

29. Yamamoto NH, Yamaguchi K, Ohmura T, et al. Serum milk fat globule epidermal growth factor 8 elevation may subdivide systemic lupus erythematosus into two pathophysiologically distinct subsets. Lupus 2014; 23: 386-394.

30. Aziz MM, Ishihara S, Rumi MA, et al. Prolactin induces MFG-E8 production in macrophages via transcription factor C/EBP beta-dependent pathway. Apoptosis 2008; 13: 609-620. 